\title{
Apolipoprotein E-Containing Lipoproteins Protect Neurons from Apoptosis via a Signaling Pathway Involving Low-Density Lipoprotein Receptor-Related Protein-1
}

\author{
Hideki Hayashi, ${ }^{1,2}$ Robert B. Campenot, ${ }^{3}$ Dennis E. Vance, ${ }^{1,4}$ and Jean E. Vance ${ }^{1,2}$ \\ ${ }^{1}$ Group on Molecular and Cell Biology of Lipids and Departments of ${ }^{2}$ Medicine, ${ }^{3}$ Cell Biology, and ${ }^{4}$ Biochemistry, University of Alberta, Edmonton, Alberta, \\ Canada T6G 2S2
}

\begin{abstract}
Apolipoprotein E (apoE)-containing lipoproteins (LPs) are secreted by glia and play important roles in lipid homeostasis in the CNS. Glia-derived LPs also promote synaptogenesis and stimulate axon growth of CNS neurons. Here, we provide evidence that glia-derived LPs protect CNS neurons from apoptosis by a receptor-mediated signaling pathway. The protective effect was greater for apolipoprotein E3 than for apolipoprotein E4, the expression of which is a risk factor for Alzheimer's disease. The anti-apoptotic effect of LPs required the association of apolipoprotein E with lipids but did not require cholesterol. Apoptosis was not prevented by lipids alone or by apoA1- or apoJ-containing lipoproteins. The prevention of neuronal apoptosis was initiated after the binding of LPs to the low-density lipoprotein receptor-related protein (LRP), a multifunctional receptor of the low-density lipoprotein receptor family. We showed that inhibition of LRP activation, by treatment of neurons with receptor-associated protein or anti-LRP antibodies, or by LRP gene-silencing experiments, reduced the protective effect of LPs. Furthermore, another LRP ligand, $\alpha_{2}$-macroglobulin, also protected the neurons from apoptosis. After binding to LRP, LPs initiate a signaling pathway that involves activation of protein kinase $\mathrm{C} \delta$ and inactivation of glycogen synthase kinase- $3 \beta$. These findings indicate the potential for using glial lipoproteins or an activator of the LRP signaling pathway for treatment for neurodegenerative disorders such as Alzheimer's disease.
\end{abstract}

Key words: apolipoprotein E; apoptosis; glia; lipoproteins; low-density lipoprotein receptor-related protein; glycogen synthase kinase-3 $\beta$

\section{Introduction}

Cholesterol is highly enriched in the brain compared with peripheral tissues, and almost all cholesterol in the brain is synthesized in situ in the CNS (Dietschy and Turley, 2004). Although the delivery of plasma lipoproteins from the circulation to the CNS is precluded by the blood-brain barrier, the CNS contains a distinct population of high-density lipoproteins that are secreted by glia and contain apolipoprotein E (apoE) as their major apolipoprotein (Han, 2004). ApoE is thought to play an important role in the CNS, because apoE synthesis increases dramatically after injury of either the CNS or peripheral nervous system (Ignatius et al., 1986; Mahley, 1988). In addition, neurodegeneration is increased by apoE deficiency during aging (Masliah et al., 1995). Furthermore, the $\varepsilon 4$ allele of apoE is the strongest known genetic risk factor for development of late-onset Alzheimer's disease (Strittmatter et al., 1993). Based on these and other observations, apoE has been proposed to contribute to the repair and/or protection of neurons from injury, although the mechanisms

Received May 18, 2006; accepted Jan. 9, 2007.

This work was supported by a grant from the Canadian Institutes for Health Research. D.E.V. is a scientist of the Alberta Heritage Foundation for Medical Research and holder of the Canada Research Chair in Molecular and Cell Biology of Lipids. We thank Russell Watts, Priscilla Gao, and Audric Moses for excellent technical assistance.

Correspondence should be addressed to Jean E. Vance, 332 Heritage Medical Research Centre, University of Alberta, Edmonton, Alberta, Canada T6G 2S2. E-mail: jean.vance@ualberta.ca.

DOI:10.1523/JNEUROSCI.5471-06.2007

Copyright $\odot 2007$ Society for Neuroscience $\quad$ 0270-6474/07/271933-09\$15.00/0 involved are unknown. ApoE is a ligand for receptors of the lowdensity lipoprotein receptor (LDLR) superfamily, several of which act both as endocytic receptors (Brown and Goldstein, 1976) and signaling receptors (Herz and Bock, 2002). Some of these receptors, including the multifunctional LDLR-related protein (LRP), are expressed in neurons in the CNS (Zhuo et al., 2000).

In addition to providing nutrient support for neurons, glial cells are thought to deliver apoE-containing lipoproteins (LPs) to neurons and to play an active role in synaptogenesis (Mauch et al., 2001) and axon growth (Hayashi et al., 2004). A loss of neurons by apoptosis is characteristic of many neurodegenerative disorders such as Alzheimer's disease, cerebral ischemia, and Niemann-Pick type C disease. In this study, we provide the first evidence that glial LPs protect CNS neurons from apoptosis and that the protection is mediated by an LRP-mediated signaling pathway in which protein kinase $\mathrm{C} \delta(\mathrm{PKC} \delta)$ is activated and the proapoptotic kinase glycogen synthase kinase-3 $\beta$ (GSK3 $\beta$ ) (Linseman et al., 2004) is inactivated.

\section{Materials and Methods}

Materials. A rabbit polyclonal antibody (R2629) directed against human LRP was generously provided by Dr. D. K. Strickland (University of Maryland School of Medicine, Baltimore MD) (Kounnas et al., 1993). Rabbit polyclonal antibodies directed against the rat LDLR were provided by Dr. G. C. Ness (University of South Florida, Tampa, FL). A cDNA construct encoding human receptor-associated protein (RAP) as a 
glutathione $S$-transferase (GST) fusion protein was a gift from Dr. Z. Yao (University of Ottawa Heart Institute, Ottawa, Ontario, Canada). cDNAs encoding human apoE3 and apoE4, as glutathione $S$-transferase fusion proteins, were generously provided by Dr. C. L. Wellington (University of British Columbia, Vancouver, British Columbia, Canada). Recombinant apoE3 and apoE4 were purified using a GSTrap FF column and Precision Protease (GE Healthcare, Uppsala, Sweden). Recombinant human apoE3 (used for the experiments depicted in Fig. $2 \mathrm{~A}$ ) was purchased from Panvera (Madison, WI). RAP was purified using a GSTrap FF column, thrombin, and a HiTrap Benzamidine FF column (GE Healthcare). Human apoA1 was prepared from human plasma (Choi et al., 2003) and was provided by Dr. G. A. Francis (University of Alberta, Edmonton, Alberta, Canada). A colony of $A p o E^{-/-}$mice was established at the University of Alberta from mice obtained from The Jackson Laboratory (Bar Harbor, ME). $\alpha_{2}$-Macroglobulin was purchased from Sigma (St. Louis, $\mathrm{MO})$.

Isolation and culture of retinal ganglion cells. Primary cultures of rat retinal ganglion cells (RGCs) were prepared from 2-d-old Sprague Dawley rats according to the procedure of Barres et al. (1988) with minor modifications. The RGCs were suspended in basal medium containing "trophic additives" [defined herein as forskolin (10 $\mu \mathrm{M}$; Sigma), brainderived neurotrophic factor (50 ng/ml; PeproTech, Rocky Hill, NJ), ciliary neurotrophic factor $(50 \mathrm{ng} / \mathrm{ml}$; PeproTech), basic fibroblast growth factor (PeproTech), and B27 supplement (Invitrogen, Carlsbad, CA)] (Barres et al., 1988). Basal medium contained glutamine (1 mM), insulin $(5 \mu \mathrm{g} / \mathrm{ml}), N$-acetylcysteine $(60 \mu \mathrm{g} / \mathrm{ml})$, progesterone $(62 \mathrm{ng} / \mathrm{ml})$, putrescine $(16 \mu \mathrm{g} / \mathrm{ml})$, sodium selenite $(40 \mathrm{ng} / \mathrm{ml})$, bovine serum albumin $(0.1 \mathrm{mg} / \mathrm{ml})$, triiodothyronine $(40 \mathrm{ng} / \mathrm{ml})$, transferrin $(0.1 \mathrm{mg} / \mathrm{ml})$, and sodium pyruvate $(1 \mathrm{~mm})$ in Neurobasal medium (Invitrogen). RGCs were plated at a density of 5000 cells per well on 96-well culture plates coated with poly-D-lysine (Sigma) and laminin (Sigma) and were cultured for 6-14 d before the start of experiments.

Culture of cortical glial cells. Glial cells were isolated from the cerebral cortex of 1-d-old rats (Gong et al., 2002) and were maintained in DMEM containing $10 \%$ fetal bovine serum. The cell preparation was highly enriched (>90\%) in astrocytes (Hayashi et al., 2004).

Isolation of lipoproteins from glia-conditioned medium. Glial cells were washed three times with PBS. Basal medium (the same as used for culture of RGCs but lacking brain-derived neurotrophic factor, ciliary neurotrophic factor, fibroblast growth factor, forskolin, and B27 supplement) was added for $3 \mathrm{~d}$. Glial cell-conditioned medium was collected and then centrifuged at room temperature for $10 \mathrm{~min}$ at $1000 \times \mathrm{g}$. The supernatant, designated glia-conditioned medium, was subjected to ultracentrifugation (Hayashi et al., 2004) on discontinuous sucrose gradients consisting of sucrose solutions of densities $1.30,1.20,1.10$, and $1.006 \mathrm{~g} / \mathrm{ml}$ in a Beckman SW40-Ti (Beckman Coulter, Fullerton, CA) rotor at $4^{\circ} \mathrm{C}$ for $48 \mathrm{~h}$ at $160,000 \times \mathrm{g}$. Twelve fractions were collected sequentially from the top of the tube and immunoblotted for apolipoproteins as described below. Fractions of densities 1.07-1.12 g/ml were combined and concentrated on an Amicon (Beverly, MA) Ultra filter (100 kDa molecular weight cutoff; Millipore, Bedford, MA). Cholesterol was extracted from the fractions twice with hexane/isopropanol $(3: 2, \mathrm{v} / \mathrm{v})$, the solvents were evaporated, and then bis(trimethylsilyl)trifluoroacetamide was added for $20 \mathrm{~min}$ at $50^{\circ} \mathrm{C}$ to generate the trimethylsilyl derivative of cholesterol for analysis by gas-liquid chromatography (Hayashi et al., 2004).

Immunoblotting. For immunoblotting of signaling molecules, RGCs were harvested on ice in sample buffer containing $62.5 \mathrm{~mm}$ Tris- $\mathrm{HCl}, \mathrm{pH}$ $6.8,10 \%$ glycerol, $2 \%$ SDS, and $5 \% \beta$-mercaptoethanol, $9 \mathrm{~h}$ after the induction of apoptosis. For immunoblotting of apolipoproteins, lipoprotein fractions were mixed with an equal volume of twiceconcentrated sample buffer. Samples were boiled for $5 \mathrm{~min}$, and proteins were separated by $0.1 \%$ SDS-PAGE and then transferred to polyvinylidene difluoride membranes at $65 \mathrm{~V}$ for $2 \mathrm{~h}$ at $4^{\circ} \mathrm{C}$. The membranes were incubated with $5 \%$ nonfat milk or $5 \%$ bovine serum albumin in $10 \mathrm{~mm}$ Tris- $\mathrm{HCl}, \mathrm{pH} 7.4$, containing $0.9 \% \mathrm{NaCl}$ and $0.1 \%$ Tween 20 for $1 \mathrm{~h}$ at room temperature. The membranes were then incubated overnight at $4^{\circ} \mathrm{C}$ with primary antibodies in $10 \mathrm{~mm}$ Tris, $\mathrm{pH} 7.4$, containing $0.9 \%$ $\mathrm{NaCl}, 0.1 \%$ Tween 20 , and $5 \%$ bovine serum albumin, and subsequently incubated with horseradish peroxidase-conjugated mouse anti-goat IgG (dilution, 1:5000; Pierce Biotechnology, Rockford, IL), horseradish peroxidase-conjugated goat anti-mouse IgG (dilution, 1:2000; Pierce Biotechnology), or horseradish peroxidase-conjugated goat anti-rabbit IgG (dilution, 1:2000; Pierce Biotechnology) for $1 \mathrm{~h}$ at room temperature. Immunoreactive proteins were detected by enhanced chemiluminescence (GE Healthcare), Super Signal West Dura (Pierce Biotechnology), or West Femto (Pierce Biotechnology). The following antibodies were used: rabbit anti-rat LDLR (dilution, 1:1000), mouse anti-human lipoprotein receptor 11 (LR11; dilution, 1:1000; BD Biosciences, San Jose, CA), goat anti-human apoE receptor 2 (apoER2; dilution, 1:400; Santa Cruz Biotechnology, Santa Cruz, CA), goat anti-human LRP (dilution, 1:1000; Santa Cruz Biotechnology), rabbit anti-human apoA1 (dilution, 1:10,000; Merck Chemicals, Darmstadt, Germany), goat antihuman apoE (dilution, 1:5000; Biodesign, Saco, ME), goat anti-human apoJ (dilution, 1:2500; Rockland Immunochemicals, Gilbertsville, PA), rabbit anti-human GSK3 $\beta$ and phospho(Ser-9)-GSK3 $\beta$ (dilution, 1:1000; Cell Signaling Technology, Danvers, MA), mouse antiDrosophila GSK3 and phospho(Tyr279/Tyr216)-GSK3 (dilution, 1:500; Millipore), rabbit anti-mouse signal transducer and activator of transcription 3 (STAT3) and phospho(Tyr-705)-STAT3 (dilution, 1:1000; Cell Signaling Technology), rabbit anti-mouse Akt and phospho(Ser473)-Akt (dilution, 1:1000; Cell Signaling Technology), rabbit antihuman p38 mitogen-activated protein kinase (MAPK) and phospho(Thr-180/Tyr-182)-p38 MAPK (dilution, 1:1000; Cell Signaling Technology), mouse anti-rat p44/42 MAPK and phospho(Thr-202/Tyr204)-p44/42 MAPK (dilution, 1:1000; Cell Signaling Technology), rabbit anti-human c-Jun N-terminal protein kinase (JNK) and phospho(Thr183/Tyr-185)-JNK (dilution, 1:1000; Cell Signaling Technology), and mouse anti- $\beta$-actin (dilution, 1:10,000; Sigma).

Induction of apoptosis in RGCs and incubation of RGCs with LPs. Apoptosis was induced in RGCs by withdrawal of trophic additives that support RGC survival (brain-derived neurotrophic factor, ciliary neurotrophic factor, basic fibroblast growth factor, forskolin, and B27 supplement) from the culture medium. RGCs were washed three times with $150 \mu \mathrm{l} /$ well basal medium lacking these trophic additives [BM $(-)]$ and then incubated with $100 \mu \mathrm{l}$ of $\mathrm{BM}(-)$ for $2 \mathrm{~h}$ at $37^{\circ} \mathrm{C}$. In some experiments, as indicated, $\mathrm{BM}(-)$ was supplemented with protein kinase inhibitors, antibodies, or RAP. The culture medium was then changed to medium (100 $\mu \mathrm{l} /$ well $)$ that contained the indicated supplements, such as LPs and/or methylamine-activated $\alpha_{2}$-macroglobulin (100 nм). $\alpha_{2}$ Macroglobulin was activated by treatment with $100 \mathrm{~mm}$ methylamine for $1 \mathrm{~h}$ at room temperature (Bacskai et al., 2000).

Detection of apoptotic RGCs with Hoechst dye or annexin V. For detection of apoptosis, RGCs were stained with Hoechst 33258. The neurons were washed twice with $100 \mu \mathrm{l}$ of PBS per well and then fixed by incubation for 15 min with $4 \%$ paraformaldehyde. Cells were washed with 100 $\mu \mathrm{l}$ of PBS per well, incubated with Hoechst dye ( $1 \mu \mathrm{g} / \mathrm{ml}$; Invitrogen $)$ for $15 \mathrm{~min}$ at room temperature, and then washed twice with $100 \mu \mathrm{l} /$ well PBS. Fluorescence was detected using a Leica DM IRE2 fluorescence microscope (Leica Microsystems, Bannockburn, IL). Stained nuclei of RGCs were counted on a monitor connected to the microscope. Neurons containing fragmented or shrunken nuclei were scored as apoptotic. Data indicate the number of apoptotic neurons as a percentage of total number of neurons. For detection of apoptosis with annexin V-fluorescein and propidium iodide, instructions from the manufacturer (Roche Diagnostics, Penzberg, Germany) were followed, and images were viewed with a Leica DM IRE2 fluorescence microscope (Leica Microsystems). Annexin V binds phosphatidylserine that becomes exposed on the outer leaflet of the plasma membrane during the early stages of apoptosis. Propidium iodide is a membrane-impermeable reagent that binds DNA and stains nuclei of necrotic cells and cells in the end stages of apoptosis. Normal living cells do not stain with either reagent, whereas cells in the early stages of apoptosis stain with annexin $\mathrm{V}$-fluorescein but not propidium iodide, and cells in the late stages of apoptosis, necrotic cells, and dead cells stain with both annexin $\mathrm{V}$ and propidium iodide.

LRP-1 small interfering RNA transfection. Control small interfering RNA (siRNA; AllStars Negative siRNA-fluorescein) and two siRNAs (designated LRP \#1 and LRP \#2, catalog numbers SI01630055 and SI01630062, respectively) specific for LRP-1 were purchased from Qia- 
A

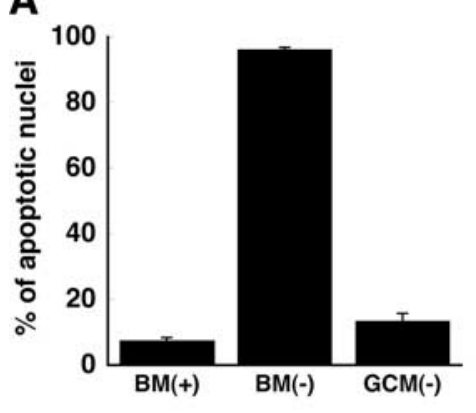

B
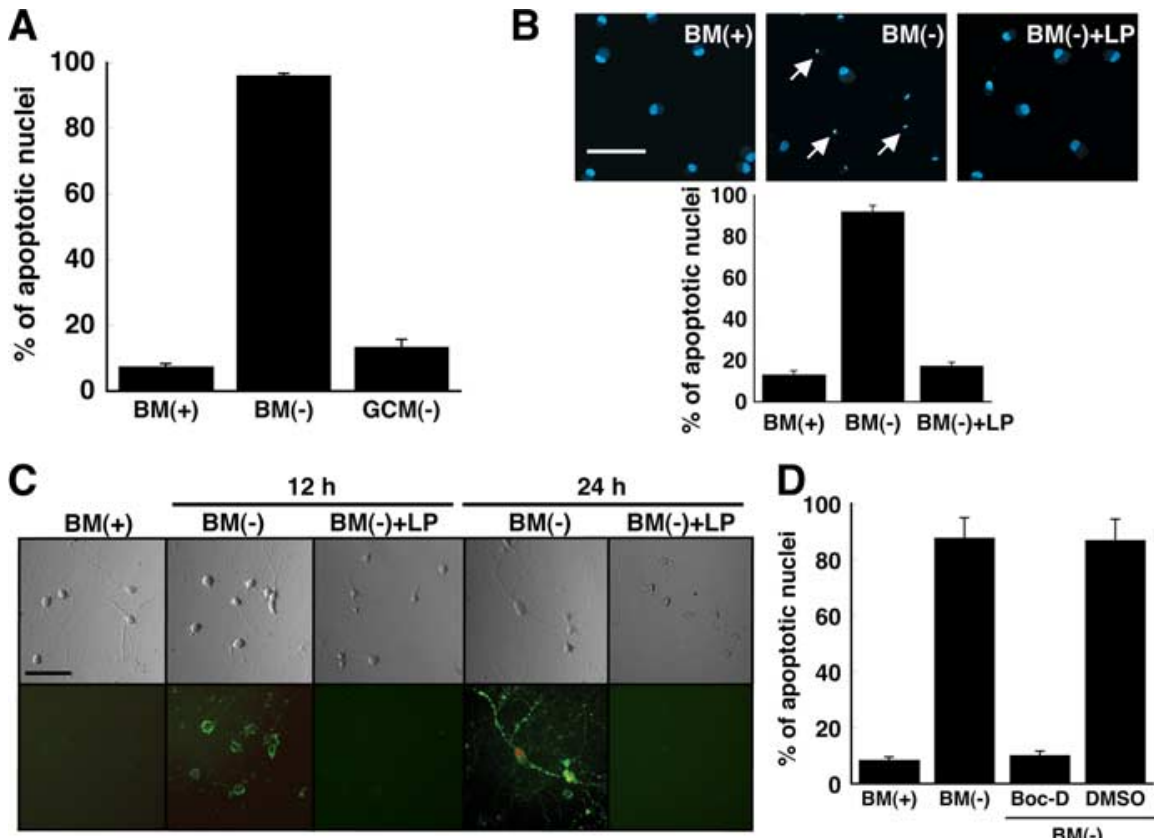

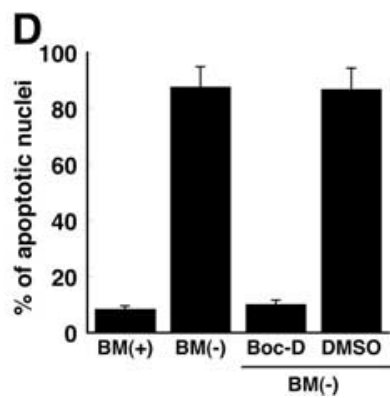

$B M(-)$

Figure 1. ApoE-containing glial lipoproteins prevent neuronal apoptosis induced by withdrawal of trophic additives. $\boldsymbol{A}, \boldsymbol{B}$, Fragmented/shrunken nuclei were detected by Hoechst staining of RGCs after incubation for $24 \mathrm{~h}$ in $B M(+)$ ] or $B M(-)$. Gliaconditioned medium without trophic additives $[\mathrm{GCM}(-)]$ or basal medium without trophic additives but with apoE-containing $\mathrm{LPs}$ was added to RGCs. The final cholesterol concentration of LPs in the media was $1 \mu \mathrm{g} / \mathrm{ml}$. The number of apoptotic neurons was quantified as a percentage of the total number of neurons. Data are means \pm SE from five independent experiments. $\boldsymbol{B}$, Typical images of Hoechst-stained RGCs are shown. Typical apoptotic nuclei are indicated by arrows. C, Shown are phase-contrast (top) and fluorescence (bottom) images of RGCs stained with annexin V-fluorescein (green) and propidium iodide (red) after incubation for 12 and $24 \mathrm{~h}$ under the same conditions as for $\boldsymbol{B}$. Representative images are shown from one of three independent experiments with similar results. Scale bars: $B, C, 50 \mu \mathrm{m}$. D, Apoptosis induced by withdrawal of trophic additives is inhibited by a broadspectrum caspase inhibitor. $\mathrm{RGCS}$ were incubated for $24 \mathrm{~h}$ in $\mathrm{BM}(+)$ or $\mathrm{BM}(-)$. The medium added to some cultures contained the broad-spectrum caspase inhibitor Boc-D fmk (Boc-D) $(100 \mu \mathrm{m})$ dissolved in dimethylsulfoxide. Medium provided to control cells (DMSO) contained an equivalent amount $(2 \mu \mathrm{l} / \mathrm{ml})$ of dimethylsulfoxide without inhibitor. Apoptotic neurons were detected by Hoechst staining. Data are means \pm SE from four independent experiments.

gen (Mississauga, Ontario, Canada). RGCs that had been cultured for 3-4 d were transfected with 20 pmol of siRNA (control or LRP-1) using the GeneSilencer siRNA Transfection Reagent (Gene Therapy Systems, San Diego, CA) according to the manufacturer's instructions. In brief, siRNA and GeneSilencer were separately diluted in OptiMEM (Invitrogen) and then mixed and incubated for $10 \mathrm{~min}$ at room temperature. The mixture was incubated with RGCs for $3 \mathrm{~d}$. Transfected RGCs were collected for immunoblotting or apoptosis experiments, which were performed as described above.

Reconstituted apoE-containing high-density lipoproteins. Recombinant human apoE3 and apoE4 were prepared as described previously (Mabile et al., 2003; Hirsch-Reinshagen et al., 2004) and reconstituted into highdensity lipoproteins (rHDLs) according to Li et al. (2002) and Thuahnai et al. (2001). The molar ratio of 1-palmitoyl-2-oleoyl-glycerophosphocholine:cholesterol:apoE in the reconstituted particles was either 100: $10: 1$ or $100: 0: 1$, as indicated. Briefly, $2.71 \mathrm{mg}$ of 1-palmitoyl-2-oleoylglycerophosphocholine (Sigma) with or without $0.14 \mathrm{mg}$ of cholesterol (Sigma) in chloroform was placed in a glass tube, and the solvent was evaporated under a stream of nitrogen. Tris- $\mathrm{HCl}(400 \mu \mathrm{l} ; 10 \mathrm{~mm}), \mathrm{pH}$ 7.4 , containing $0.9 \% \mathrm{NaCl}$, was added, and the suspension was incubated for $1 \mathrm{~h}$ on ice with vortexing for $30 \mathrm{~s}$ every $15 \mathrm{~min}$. Sodium cholate (final concentration, $3 \mathrm{mg} / \mathrm{ml}$ ) was added, and the mixture was incubated for $2 \mathrm{~h}$ on ice, by which time the solution became translucent. Recombinant apoE3 or apoE4 $(1 \mathrm{mg})$ was added, and the mixture was incubated for $1 \mathrm{~h}$ on ice. To this solution, we added $100 \mathrm{mg}$ of Bio-Beads (Bio-Rad, Hercules, CA) that had been equilibrated according to manufacturer's instructions in $10 \mathrm{~mm}$ Tris- $\mathrm{HCl}, \mathrm{pH} 7.4$, containing $0.9 \% \mathrm{NaCl}$. The mixture was gently mixed by rotation for $3 \mathrm{~h}$ at $4^{\circ} \mathrm{C}$. The solution containing the beads was removed with a syringe, filtered using a $0.45 \mu \mathrm{m}$ sterile filter, and subjected to sucrose density gradient centrifugation as described above for isolation of glia-derived LPs. ApoE-containing fractions (density, $1.07-1.21 \mathrm{~g} / \mathrm{ml}$ ) were collected and combined.

\section{Results \\ ApoE-containing lipoproteins protect neurons from apoptosis}

Rat RGCs were chosen for the studies because they are CNS neurons that can be cultured with a high degree of purity. Apoptosis of RGCs was induced by withdrawal of growth supplements (brainderived neurotrophic factor, ciliary neurotrophic factor, fibroblast growth factor, B27 supplement, and forskolin) from the culture medium. These additives support RGC survival, and we term them collectively trophic additives. Because each of these components contributes to neuronal survival, we investigated neuronal survival after simultaneous elimination of all of these additives from the medium. Twenty-four hours after removal of the trophic additives, Hoechst staining revealed that $>90 \%$ of the neurons contained shrunken or fragmented nuclei, which are markers of apoptosis (Fig. $1 A, B)$. Withdrawal of each trophic additive individually from the culture medium did not result in significant numbers of apoptotic nuclei after $24 \mathrm{~h}$ (data not shown). Exposure of phosphatidylserine on the surface of the neurons (another marker of apoptosis) $12 \mathrm{~h}$ after withdrawal of trophic additives was monitored by the binding of annexin $\mathrm{V}$-fluorescein in the absence of propidium iodide staining (Fig. 1C). In addition, the broad-spectrum caspase inhibitor Boc-D fmk prevented neuronal death (Fig. 1D), implying that withdrawal of trophic additives from RGC culture medium induced caspase-dependent apoptosis.

Medium conditioned by primary cultures of rat cortical glia ( $>90 \%$ astrocytes) for $3 \mathrm{~d}$ was supplied to RGCs for $24 \mathrm{~h}$ in the absence of trophic additives. The glia-conditioned medium prevented apoptosis of the neurons (Fig. 1A). Because apoE and lipoprotein receptors have been implicated in signaling pathways in the brain (Herz and Bock, 2002), we determined whether gliaderived LPs present in conditioned medium were active in preventing apoptosis. Glial LPs were isolated from conditioned medium by ultracentrifugation and supplied in basal medium to RGCs at concentrations of cholesterol and apoE equivalent to those in glia-conditioned medium $(\sim 1 \mu \mathrm{g} / \mathrm{ml}$ cholesterol and $3-5 \mu \mathrm{g} / \mathrm{ml}$ apoE) (Hayashi et al., 2004). Figure 1, $B$ and $C$, shows that LPs secreted by glia prevented apoptosis of RGCs. Neurons that were given LPs did not stain with annexin V-fluorescein or propidium iodide after $24 \mathrm{~h}$ and were, therefore, not apoptotic. However, the majority of RGCs cultured in basal medium lacking trophic additives were annexin $\mathrm{V}$ and propidium iodide positive after $24 \mathrm{~h}$ (Fig. 1C), indicating that these neurons were in the late stages of apoptosis.

The addition of recombinant human apoE alone, cholesterol alone, or a mixture of cholesterol and recombinant apoE3 in 
amounts equivalent to those in gliaconditioned medium, failed to prevent neuronal apoptosis (Fig. 2A). To determine whether or not apoE was required for protection against apoptosis, lipoproteins that contained or lacked apoE were isolated from the conditioned medium of glia cultured from $A p o E^{+/+}$and $A p o E^{-/-}$ mice, respectively (Fig. $2 B$ ). The lipoproteins $(120 \mu \mathrm{g}$ of protein $/ \mathrm{ml})$ were supplied to RGCs in basal medium, and apoptosis was assessed. Lipoproteins isolated from $A p o E^{-/-}$glia failed to prevent apoptosis of the RGCs, whereas lipoproteins secreted by $A p o E^{+/+}$glia protected the neurons from apoptosis (Fig. 2C). Because the conditioned medium from $A p o E^{-/-}$glia contained only a small amount of cholesterol compared with that in $A p o E^{+/+}$glial conditioned medium, we wished to determine whether the failure of lipoproteins from $A p o E^{-/-}$glia to protect against apoptosis was caused by a deficiency of cholesterol in the lipoprotein particles. We therefore generated lipoproteins lacking apoE but containing apoAl by incubation of primary cultures of cortical glia from $A p o E^{-/-}$mice with human apoA1. The apoA1-containing lipoproteins contained the same amount of cholesterol $(1 \mu \mathrm{g} / \mathrm{ml})$ and were of the same density as the apoEcontaining lipoproteins but contained apoA1 instead of apoE (Fig. 2 B). The addition of apoA1-containing lipoproteins to RGCs failed to protect the neurons from apoptosis (Fig. 2D). Because the apoJ content of the apoE- and apoA1containing lipoprotein fractions was similar (Fig. $2 B$ ), the apoJ-containing lipoproteins from $A p o E^{+/+}$and $A p o E^{-/-}$glia could not have imparted the protective effect. These observations demonstrate that apoE is required for preventing apoptosis of the RGCs and that the protective effect of apoE requires its association with lipid.

To confirm that the apoE component of LPs was responsible for the protection of RGCs against apoptosis, recombinant apoE was generated and reconstituted into rHDLs that contained phospholipids and cholesterol (molar ratio of apoE:1-palmitoyl2-oleoyl-glycerophosphocholine:cholesterol of 1:100:10). When these rHDLs were supplied to RGCs, so that the amount of apoE was equivalent to that in glia-derived LPs, apoptosis was prevented (Fig. $2 E$ ). In contrast, liposomes that had the same lipid composition but lacked apoE did not protect the neurons from apoptosis (Fig. 2 E). To determine whether the protection against apoptosis was mediated by cholesterol in the rHDLs, we prepared rHDLs that contained 1-palmitoyl-2-oleoyl-glycerophosphocholine and apoE (molar ratio of 100:1) but did not contain cholesterol. The rHDLs that lacked cholesterol were as effective in preventing apoptosis as were the rHDLs that contained cholesterol (Fig. $2 F$ ). These observations demonstrate that the antiapoptotic effect of glial lipoproteins requires apoE that is associated with lipid but that cholesterol is not required. experiments. chol, Cholesterol.
B
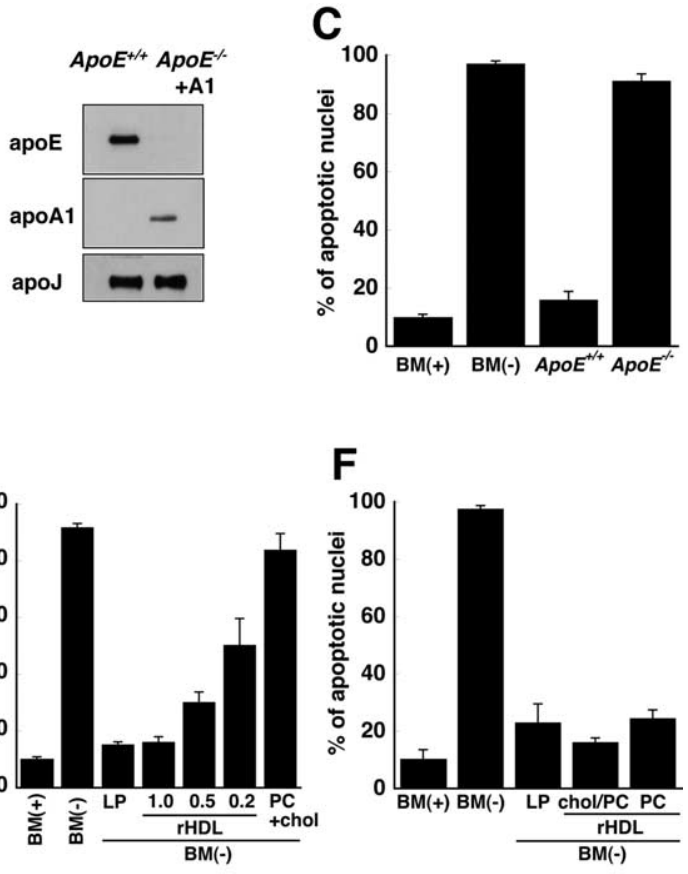

Figure 2. ApoE-containing lipoproteins, but not apoE alone, cholesterol alone, or apoA1/apoJ-containing lipoproteins, prevent apoptosis of RGCs. $\boldsymbol{A}$, Recombinant human apoE3 and/or cholesterol (5 and $1 \mu \mathrm{g} / \mathrm{ml}$, respectively) were added to $\mathrm{BM}(-)$ and

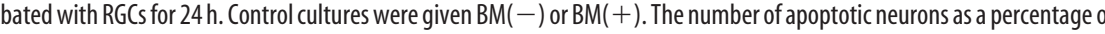
列 列 mount of apoE as in LPs and either contained (chol/PC) or lacked (PC) cholesterol. For all experiments, fragmented/shrunken nuclei were detected by Hoechst staining and designated as apoptotic cells. Data are means $\pm \mathrm{SE}$ from three or four independent

Protection from apoptosis is mediated by LRP

Previous studies have demonstrated that apoE in association with lipids, but not lipid-free apoE, is a ligand for receptors of the LDLR family and that lipid-free apoE and lipid-associated apoE have different affinities for different members of the LDLR family (Innerarity et al., 1979; Ruiz et al., 2005). We therefore determined whether the protective effect of LPs was mediated by a member of this receptor superfamily. The following receptors of the LDLR family were detected in RGCs by immunoblotting: LDLR, LR11, LRP, and apoER2 (Fig. 3A).

To determine whether one of these receptors mediated the protection against apoptosis, we incubated RGCs with RAP, which is known to compete with ligands for binding to receptors of the LDLR superfamily (Innerarity et al., 1979). RAP itself did not induce apoptosis in RGCs, but the ability of LPs to protect RGCs from apoptosis was eliminated by RAP (Fig. 3B). To determine whether LRP was involved in protecting the RGCs from apoptosis, we blocked the binding of LPs to LRP by adding a polyclonal antibody directed against LRP (Kounnas et al., 1992). The antibody blocked the protective effect of LPs but did not by itself cause apoptosis (Fig. 3C). These observations suggest that LRP is involved in the LP-mediated protection of RGCs from apoptosis. LRP has multiple ligands, including $\alpha_{2}$-macroglobulin (Herz and Strickland, 2001). Thus, we incubated RGCs with $\alpha_{2^{-}}$ 
A

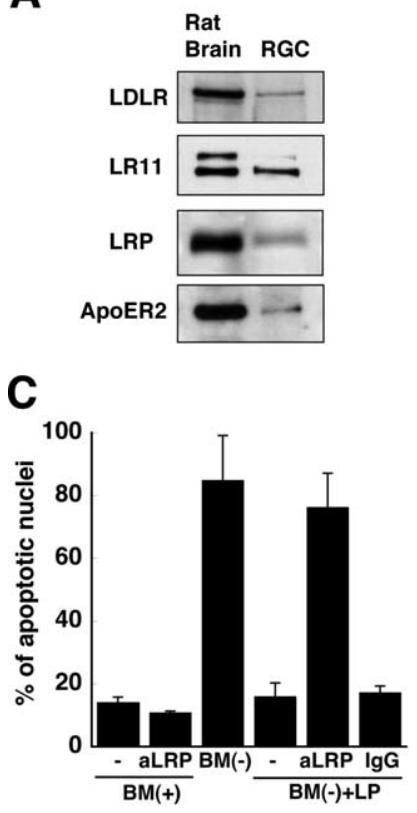

B
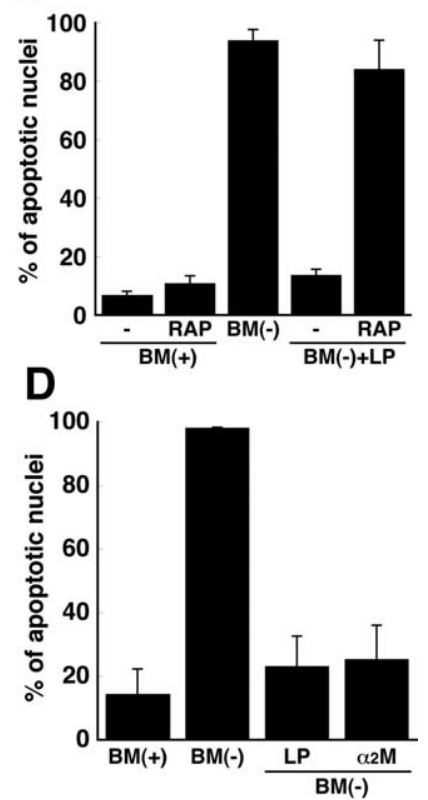

Figure 3. LRP mediates the protective effect of apoE-containing lipoproteins. $\boldsymbol{A}$, Receptors of the low-density lipoprotein receptor family were detected by immunoblotting of $20 \mu \mathrm{g}$ of protein from lysates of RGCs. Rat brain proteins $(20 \mu \mathrm{g})$ were used as a positive control. Data are from one experiment representative of three experiments with similar results. $\boldsymbol{B}-\boldsymbol{D}, \mathrm{RGCs}$ were incubated for $24 \mathrm{~h}$ with $\mathrm{BM}(+)$ or BM $(-)$ in the presence of apoE-containing LPs and/or $300 \mathrm{~nm}$ $\operatorname{RAP}(\boldsymbol{B}), 10 \mu \mathrm{g} / \mathrm{ml}$ anti-LRP antibody (aLRP) $(\boldsymbol{C})$, or $10 \mu \mathrm{g} / \mathrm{ml}$ nonspecific $\lg \mathrm{G}(\boldsymbol{C})$, after a $2 \mathrm{~h}$ pretreatment with RAP, antibodies, or $100 \mathrm{~nm} \alpha_{2}$-macroglobulin $(\alpha 2 \mathrm{M})(\boldsymbol{D})$. The percentage of cells containing apoptotic nuclei was determined by Hoechst staining. Data are means \pm SE of at least three independent experiments.

macroglobulin (100 nM). $\alpha_{2}$-Macroglobulin prevented apoptosis of the RGCs as effectively as did LPs (Fig. 3D).

As additional evidence that LRP was responsible for mediating the anti-apoptotic effect of LPs, we reduced the expression of LRP-1 in RGCs using RNA silencing. RGCs were transfected with either a nonspecific, control siRNA or with two different siRNAs specific for LRP-1. To determine whether the specific LRP-1 siRNAs (LRP \#1 and LRP \#2) reduced the expression of LRP-1 in RGCs, we performed immunoblotting experiments with antiLRP- 1 antibodies. Figure $4 A$ shows that in RGCs transfected with the LRP-1-specific siRNAs, the amount of LRP-1 protein was markedly less than in RGCs transfected with control siRNA. To determine whether the decreased expression of LRP-1 reduced the ability of LPs to protect RGCs from apoptosis, we assessed apoptosis in the siRNA-transfected neurons. Neither the LRP-1specific siRNAs nor the control siRNA induced apoptosis in RGCs cultured in the presence of trophic additives (Fig. $4 B$ ). As expected, essentially all of the RGCs cultured without LPs in the absence of trophic additives were apoptotic. In contrast, for RGCs transfected with LRP-1-specific siRNAs and cultured in the absence of trophic additives and in the presence of LPs, up to $60 \%$ of the neurons were apoptotic, whereas only $\sim 20 \%$ of RGCs transfected with control siRNA were apoptotic (Fig. 4B). Thus, decreased expression of LRP-1 reduced the ability of LPs to protect the neurons from apoptosis. The data in Figures 3 and 4 demonstrate, therefore, that glial LPs prevent apoptosis via a mechanism that involves LRP-1.

Binding of LP to LRP initiates a signaling pathway

Because LRP can initiate signaling cascades in the CNS (Herz and Bock, 2002), our observations suggested that the apoE-mediated

A

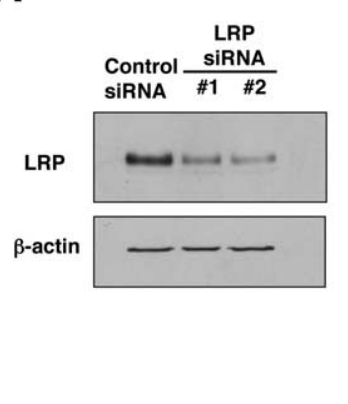

B

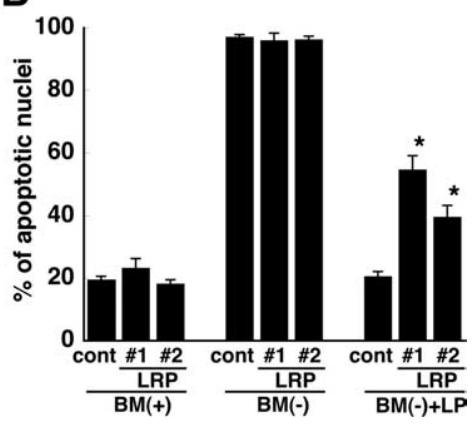

Figure 4. Reduction of LRP- 1 in RGCs by siRNA. $A$, After 3 in culture, RGCs were transfected with 20 pmol of control siRNA or two different LRP-1 siRNAs (LRP \#1 and LRP \#2). RGCs were harvested $3 \mathrm{~d}$ after transfection, and proteins were separated by $0.1 \%$ SDS-PAGE. LRP was detected by immunoblotting. Immunoblotting of $\beta$-actin was used as a loading control. Data are from one experiment that is representative of three experiments with similar results. $\boldsymbol{B}$, RGCs were transfected with control siRNA (cont) or two different siRNAs (LRP \#1 and \#2) that were specific for LRP-1. After $3 \mathrm{~d}$, the neurons were incubated for $24 \mathrm{~h}$ with $B M(+), B M(-)$, or $\mathrm{BM}(-)$ in the presence of apoE-containing lipoproteins $[\mathrm{BM}(-)+\mathrm{LP}]$. The percentage of cells containing apoptotic nuclei was determined by Hoechst staining. Data are means \pm SE of four independent experiments. ${ }^{*} p<0.0001$ for control versus LRP \#1; ${ }^{*} p=0.0003$ for control versus LRP \#2. Statistical analysis was performed using one-way ANOVA followed by Bonferroni's multiple comparison.
A
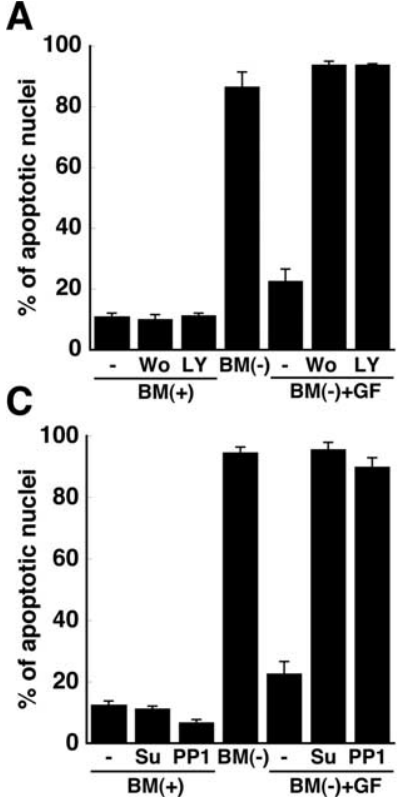

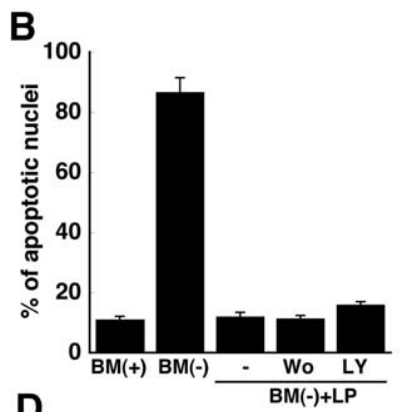

D

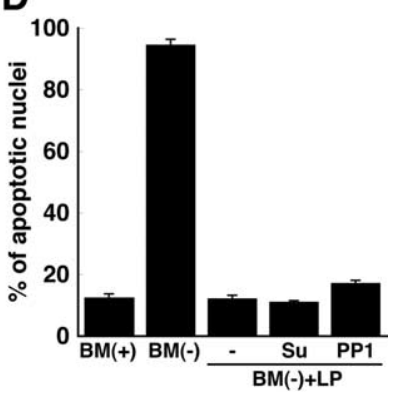

Figure 5. Phosphatidylinositol-3-kinase and Src family kinases do not contribute to the anti-apoptotic effect of apoE-containing lipoproteins. Retinal ganglion cells were incubated in $\mathrm{BM}(+), \mathrm{BM}(-)$, or $\mathrm{BM}(-)$ plus apoE-containing lipoproteins $[\mathrm{BM}(-)+\mathrm{LP}]$ for 24 h. Apoptosis was assessed by Hoechst staining. The medium added to some neuronal cultures contained brain-derived neurotrophic factor $(50 \mathrm{ng} / \mathrm{ml})$, ciliary neurotrophic factor $(50 \mathrm{ng} / \mathrm{ml})$, and forskolin (10 $\mu \mathrm{M} ;+\mathrm{GF} ; A, C$ ), and/or the phosphatidylinositol-3-kinase inhibitors wortmannin (Wo; $100 \mathrm{~nm}$ ) or LY294002 (LY; $50 \mu \mathrm{M} ; \boldsymbol{A}, \boldsymbol{B})$ or Src kinase inhibitors PP1 (10 $\mu \mathrm{m})$ or Su6656 (Su; $10 \mu \mathrm{m} ; \boldsymbol{C}, \boldsymbol{D})$. Data are means $\pm \mathrm{SE}$ of three independent experiments.

protection against apoptosis occurred via a signaling pathway that was induced after binding of lipid-associated apoE to LRP. To define this putative signaling pathway, several kinases known to participate in pathways of survival and apoptosis in neurons were examined. Src family kinases and phosphatidylinositol-3kinase mediate survival pathways in neurons induced by neurotrophic factors such as brain-derived neurotrophic factor and 
ciliary neurotrophic factor. We therefore initially tested the efficacy of inhibitors of phosphatidylinositol-3-kinase [wortmannin and 2-(4-morpholinyl)-8-phenyl4H-1-benzopyran-4-one (LY294002)] and inhibitors of Src family kinases [protein phosphatase 1 (PP1) and Su6656] in RGCs in which survival is supported by these neurotrophic factors. Although the inhibitors blocked the protective effect of the neurotrophic factors (Fig. $5 A, C$ ), these agents did not block the protective effect of glial LPs (Fig. 5B,D), implying that these kinases do not contribute to the LP-mediated protective pathway.

We also investigated whether or not the anti-apoptotic pathway involved PKC by determining whether LPs prevented neuronal apoptosis in the presence of inhibitors of PKC. 12-(2-cyanoethyl)6,7,12,13-tetrahydro-13-methyl-5-oxo$5 H$-indolo(2,3-a)pyrrolo(3,4-c)-carbazole (Gö6976) and 2-[8-[(dimethylamino) methyl]-6,7,8,9-tetrahydropyrido (1,2a)indol-3-yl]-3-(1-methylindol-3-yl)maleimide (Ro32-0432) were used as inhibitors of $\mathrm{PKC} \alpha, \mathrm{PKC} \beta$, and $\mathrm{PKC} \gamma$, whereas chelerythrine was used as an inhibitor of $\mathrm{PKC} \alpha, \mathrm{PKC} \beta, \mathrm{PKC} \gamma$, and $\mathrm{PKC} \delta$, and rottlerin was used as an inhibitor of PKC $\delta$ (Wilkinson et al., 1993; Bright and Mochly-Rosen, 2005). Chelerythrine and rottlerin abrogated the protective effect of LPs, whereas Gö6976 and Ro32-0432 did not (Fig. 6A). Importantly, chelerythrine and rottlerin did not reduce RGC survival in basal culture medium (data not shown). These data indicate that $\mathrm{PKC} \delta$ protects RGCs from apoptosis via a pathway that is initiated by LPs and LRP-1.

Alterations in the phosphorylation state of several other signaling proteins (p44/42 MAPK, p38 MAPK, STAT3, Akt, and JNK) that have been reported to participate in anti-apoptotic or proapoptotic pathways in neurons (Segal and Greenberg, 1996; Yuan and Yankner, 2000) were examined by immunoblotting (Fig. 7). No significant differences in phosphorylation levels of these proteins were detected in the presence or absence of LPs, suggesting that these kinases do not contribute to the anti-apoptotic effect of LPs. In contrast, the phosphorylation of Ser-9 of GSK3 $\beta$, a proapoptotic kinase that is inactivated by phosphorylation at Ser-9 (Linseman et al., 2004), was decreased under apoptotic conditions and was restored when RGCs were incubated with LPs (Fig. 6B,C). The phosphorylation of GSK $3 \alpha$ at Tyr-279 and GSK3 $\beta$ at Tyr-216 was not significantly changed by the presence of the lipoproteins (Fig. $6 \mathrm{~B}$ ). In support of a role of GSK in the LP-mediated increased survival of neurons, lithium, an inhibitor of GSK (Noble et al., 2005), also prevented the apoptosis in a dose-dependent manner (Fig. 6D). These data suggest that GSK3 $\beta$ contributes to the apoptosis caused by withdrawal of trophic additives from the RGCs and that LPs mediate their protective effect via a pathway that involves GSK3 $\beta$ and PKC $\delta$. Consistent with this idea, the phosphorylation of Ser-9 of GSK3 $\beta$ was reduced by RAP (Fig. $6 E$ ), a
B

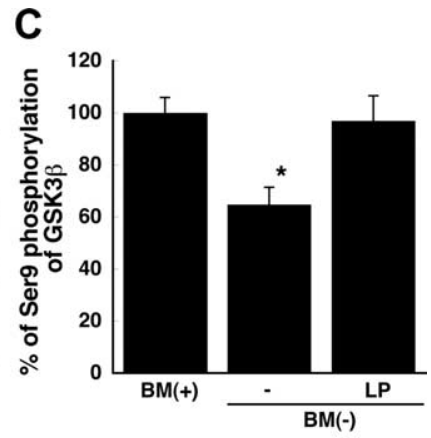

E

$\mathbf{F}$

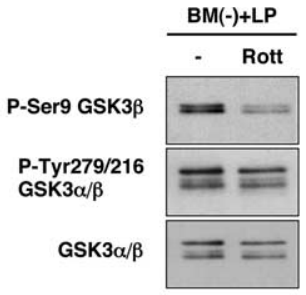

Figure 6. $\operatorname{PKC} \delta$ and GSK3 $\beta$ contribute to the anti-apoptotic effect of apoE-containing lipoproteins. $\boldsymbol{A}$, RGCs were incubated with inhibitors of PKC: Gö6976 (Go; $20 \mathrm{~nm}$ ), Ro32-0432 (Ro; $50 \mathrm{~nm}$ ), rottlerin (Rott; $2 \mu \mathrm{m})$, and chelerythrine (Chel; $1 \mu \mathrm{m})$. BM(-) was supplemented with apoE-containing LPs and PKC inhibitors and then supplied to RGCs for $24 \mathrm{~h}$ after a $2 \mathrm{~h}$ pretreatment of the 作 phorylated at Tyr-279/216 in neurons incubated as in $B$ in $B M(-)$ plus $L P$ in the absence or presence of rottlerin. Data are representative of three independent experiments with similar results.

protein that blocks the binding of LPs to LRP (Fig. 3B). Moreover, the PKC $\delta$ inhibitor rottlerin prevented the increased phosphorylation of Ser- 9 of GSK3 $\beta$ that was induced by LPs, whereas the phosphorylation of GSK $3 \alpha / \beta$ at Tyr-216/279 was unchanged (Fig. 6 F). Rottlerin did not alter the phosphorylation of Ser-9 of GSK3 $\beta$ in basal culture medium (data not shown).

\section{ApoE3 increases survival of RGCs to a greater extent than} does apoE4

In view of many reports that apoE4 is detrimental for neuronal function, we compared the efficacy of lipoproteins containing apoE3 or apoE4 in the protection of RGCs against apoptosis. Recombinant human apoE3 and apoE4 were reconstituted into rHDLs that contained phosphatidylcholine (1-palmitoyl-2oleoyl-glycerophosphocholine) (molar ratio of 1:100). Liposomes containing phosphatidylcholine alone did not protect the RGCs from apoptosis induced by withdrawal of trophic additives (Fig. 8). However, rHDLs that contained apoE3 reduced the number of apoptotic neurons from 78.0 to $24.5 \%$, whereas with apoE4-containing rHDLs, $49.8 \%$ of the RGCs were apoptotic (Fig. 8). Thus, rHDLs that contain apoE3 are more protective against apoptosis than are rHDLs that contain apoE4. 


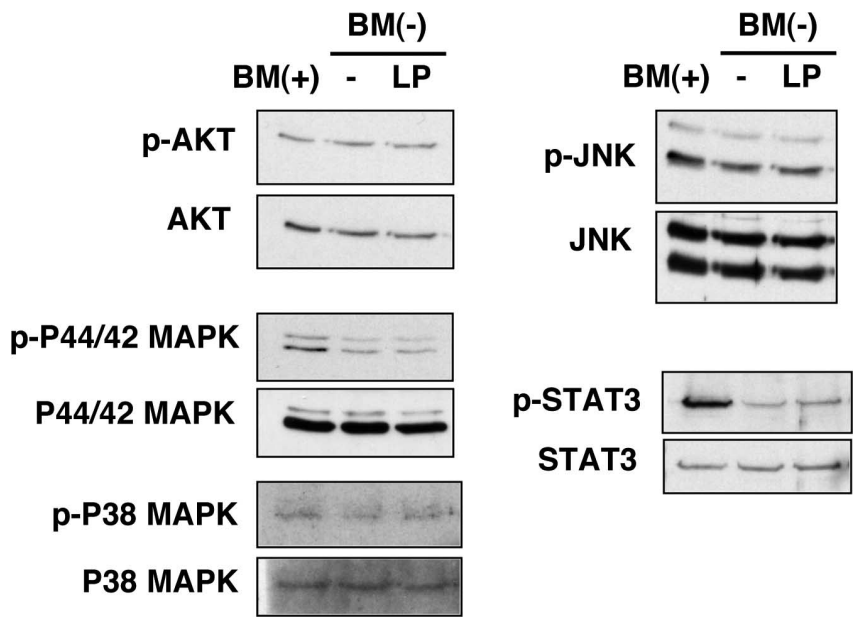

Figure 7. Phosphorylation of signaling molecules in RGCs in response to apoE-containing lipoproteins. Apoptosis was initiated in RGCs by withdrawal of trophic additives [BM(-)] from basal medium $[B M(+)]$. RGCs were incubated for $9 \mathrm{~h}$ in the presence $(\mathrm{LP})$ or absence $(-)$ of apoE-containing lipoproteins and then harvested and analyzed by immunoblotting using antibodies directed against phosphorylated (p) Akt (phosphorylated on Ser-473), P44/42 MAPK (phosphorylated on Thr-202/Tyr-204), P38 MAP kinase (phosphorylated on Thr-180/Tyr-182), STAT3 (phosphorylated on Tyr-705), and JNK (phosphorylated on Thr-183/Tyr-185). Total amounts of the proteins were detected by immunoblotting. Data are from one experiment representative of three experiments with similar results.

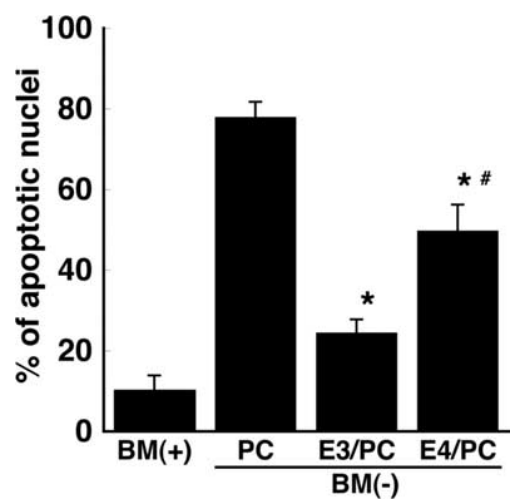

Figure 8. ApoE4-containing rHDLs are less protective against apoptosis than are apoE3containing rHDLs. rHDLs containing recombinant human apoE3 or apoE4 were prepared with phosphatidylcholine (PC) (molar ratio, 1:100). The apoE3- (E3/PC) or apoE4 (E4/PC)-containing rHDLs were added to $\mathrm{BM}(-)$ and incubated with retinal ganglion cells for $24 \mathrm{~h}$. Control cultures were given $\mathrm{BM}(+)$. Liposomes that lacked apoE but contained the same amount of $\mathrm{PC}$ as in rHDLs $(+P C)$ were used as a negative control. The number of apoptotic neurons was determined by Hoechst staining as a percentage of the total number of neurons. Data are means \pm SE from five independent experiments. ${ }^{*} p<0.005$ for $\mathrm{BM}(-)$ plus $\mathrm{PC}$ versus $\mathrm{BM}(-)$ plus $\mathrm{E} 3 / \mathrm{PC}$ and $\mathrm{BM}(-)$ plus E4/PC. $\#=0.0057$ for E3/PC versus E4/PC. Statistical analysis was performed using one-way ANOVA followed by Bonferroni's multiple comparison.

\section{Discussion}

Our studies reveal a novel function of LRP-1 that is induced in CNS neurons by glia-derived LPs: protection against apoptosis. Neuronal apoptosis is a characteristic feature of many neurodegenerative disorders, such as Alzheimer's disease and Parkinson's disease. Moreover, the levels of neurotrophic factors, such as brain-derived neurotrophic factor, in the brains of individuals with these diseases have been reported to be decreased (Siegel and Chauhan, 2000; Murer et al., 2001). Our study demonstrates that RGCs are protected from apoptosis induced by removal of trophic additives (including brain-derived neurotrophic factor and ciliary neurotrophic factor) via a signaling pathway that is initiated after binding of LPs to LRP, a receptor of the LDLR family.

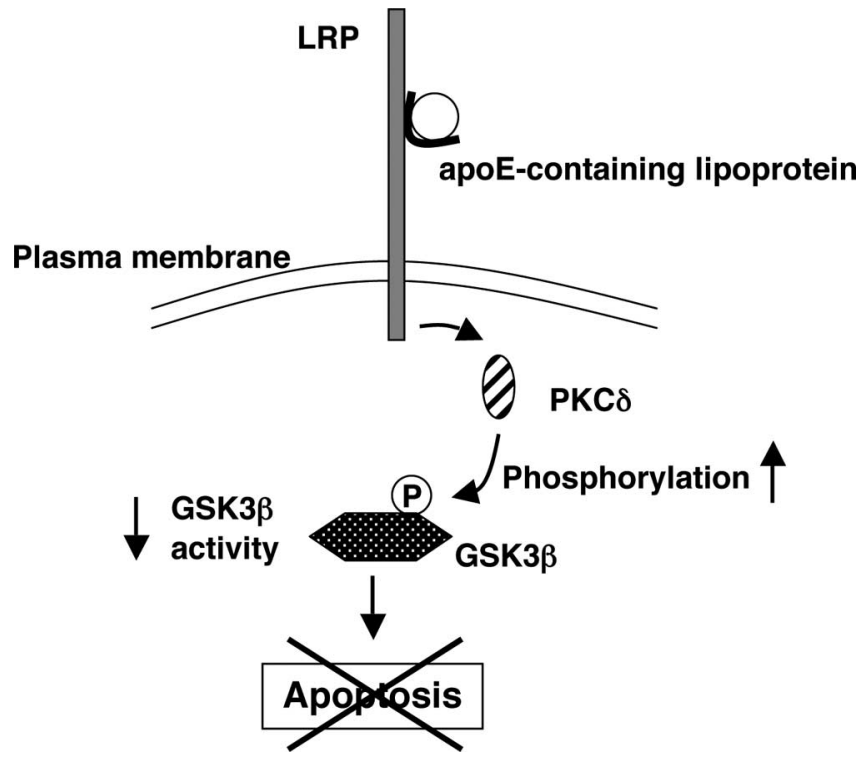

Figure 9. Proposed anti-apoptotic signaling pathway induced in RGCs by apoE-containing lipoproteins. When apoptosis is induced in RGCs by removal of trophic additives, apoEcontaining lipoproteins secreted by glia protect the RGCs from apoptosis. After binding of apoEcontaining lipoproteins to LRP on the cell surface, activation of $\operatorname{PKC} \delta$, either directly or indirectly, increases the phosphorylation (P) of GSK3 $\beta$, thereby inactivating GSK3 $\beta$ and protecting the RGCs from apoptosis.

This signaling pathway involves GSK3 $\beta$ downstream of PKC $\delta$ (Fig. 9). LRP has been reported to contribute to calcium signaling (Bacskai et al., 2000), long-term potentiation (Zhuo et al., 2000), neurite outgrowth (Holtzman et al., 1995), brain development (Herz and Chen, 2006), and Alzheimer's disease (Harris-White and Frautschy, 2005). The multifunctional receptor LRP is expressed in RGCs, the CNS neurons that we studied. ApoE is a high-affinity ligand for this receptor and shows higher affinity for LRP when the apoE is associated with lipids (Ruiz et al., 2005). Our studies demonstrate that apoE in association with lipids, but not lipid-free apoE, protects neurons from apoptosis.

Although cholesterol is regarded as an important molecule for neuronal function [e.g., cholesterol associated with apoE promotes synaptogenesis (Mauch et al., 2001), and inhibition of intracellular cholesterol trafficking causes neuronal death (Huang et al., 2006)], the anti-apoptotic effect of LPs that we observed is not mediated by cholesterol. We found that neither cholesterol alone nor cholesterol with recombinant lipid-free apoE protected the neurons from apoptosis. Furthermore, glial lipoproteins from $A p o E^{-/-}$mice did not rescue the neurons from apoptosis, nor did apoA1-or apoJ-containing lipoproteins that contained the same amount of cholesterol as LPs. However, rHDLs that contained apoE and phosphatidylcholine without any cholesterol protected the RGCs from apoptosis to the same extent as did the LPs. These observations demonstrate that the protective effect of LPs does not require cholesterol. In experiments in which we used the inhibitory protein RAP, as well as in experiments with anti-LRP antibodies and LRP-1 siRNA, we identified LRP-1 as the receptor that mediates the protective effect of LPs.

We investigated the neuronal signaling pathway that is initiated when LPs bind to LRP. Our data show that phosphorylation of Ser-9 of GSK $3 \beta$ is decreased during apoptosis and is restored by addition of LPs, suggesting that these lipoproteins protect neurons from apoptosis by inactivation of GSK3 $\beta$ caused by direct or indirect phosphorylation at Ser-9 by $\operatorname{PKC} \delta$ (Fig. 9). 
Phosphatidylinositol-3-kinase and Src family tyrosine kinases are known to play important roles in cellular survival, growth, differentiation and proliferation (Bondy and Cheng, 2004; Kuo et al., 2005). However, although inhibition of these kinases blocked the survival effects of brain-derived neurotrophic factor and ciliary neurotrophic factor in RGCs, none of these inhibitors eliminated the protective effect of LPs. Nor did MAP kinases (P44/42 and P38) appear to contribute to the protective effect of LPs, because the phosphorylation state of these kinases was not altered by LPs. Phosphorylation of the cytosolic domain of LRP has been reported to be increased by PKC $\alpha$ (Ranganathan et al., 2004). However, agents that inhibited only $\mathrm{PKC} \delta$, not $\mathrm{PKC} \alpha, \operatorname{PKC} \beta$, or PKC $\gamma$, blocked the anti-apoptotic effect of LPs in RGCs, implicating PKC $\delta$ in the signaling pathway. GSK3 $\beta$ is proapoptotic in neurons (Linseman et al., 2004), and its activity is decreased by phosphorylation at Ser-9 (Cross et al., 1995). Consistent with our observations, inactivation of GSK3 $\beta$ has been shown previously to prevent neuronal apoptosis (Mora et al., 2001). Furthermore, a GSK3 inhibitor has been shown to reduce tauopathy (Noble et al., 2005 ) and A $\beta$ production (Sun et al., 2002; Su et al., 2004), which are pathological hallmarks of Alzheimer's disease. Because the binding of LPs to LRP inactivates GSK3 $\beta$ in CNS neurons, it is possible that this pathway is a potential target for treatment of Alzheimer's disease.

ApoE appears to play important roles in the nervous system. For example, apoE synthesis in glia is dramatically increased in response to nerve injury in the CNS and peripheral nervous system (Ignatius et al., 1986; Petegnief et al., 2001). CNS neurons are more susceptible to ischemic injury in $A p o E^{-/-}$mice than in $A p o E^{+/+}$mice (Sheng et al., 1999). ApoE deficiency in mice also reduces the stability of the neuronal cytoskeleton during the aging process (Masliah et al., 1995). In addition, persistent nerve degeneration has been observed in injured brains of $A p o E^{-/-}$but not $A p o E^{+/+}$mice (Fagan et al., 1998), suggesting that apoE plays a role in the repair and/or protection of neurons.

The mechanism by which apoE is involved in Alzheimer's disease is not clear (Goedert and Spillantini, 2006). In a mouse model of Alzheimer's disease, the presence of apoE3 decreased $\mathrm{A} \beta$ levels and deposition (DeMattos, 2004; DeMattos et al., 2004). We now report that LPs secreted by glia contribute to the survival of CNS neurons by a novel mechanism that requires LRP-1. Furthermore, our data show that lipoproteins that contain apoE3 protect RGCs from apoptosis, whereas lipoproteins that contain apoE4 are significantly less protective. Our observations might provide at least a partial explanation for why inheritance of the $\varepsilon 4$, rather than the $\varepsilon 3$, allele markedly increases the risk of developing late-onset Alzheimer's disease.

\section{References}

Bacskai BJ, Xia MQ, Strickland DK, Hyman BT (2000) The endocytic receptor protein LRP also mediates neuronal calcium signaling via $\mathrm{N}$-methylD-aspartate receptors. Proc Natl Acad Sci USA 97:11551-11556.

Barres BA, Silverstein BE, Corey DP, Chun LLY (1988) Immunological, morphological, and electrophysiological variation among retinal ganglion cells purified by panning. Neuron 1:791-803.

Bondy CA, Cheng CM (2004) Signaling by insulin-like growth factor 1 in brain. Eur J Pharmacol 490:25-31.

Bright R, Mochly-Rosen D (2005) The role of protein kinase C in cerebral ischemic and reperfusion injury. Stroke 36:2781-2790.

Brown MS, Goldstein JL (1976) Receptor-mediated control of cholesterol metabolism. Science 191:150-154.

Choi HY, Karten B, Chan T, Vance JE, Greer WL, Heidenreich RA, Garver WS, Francis GA (2003) Impaired ABCA1-dependent lipid efflux and hypoalphalipoproteinemia in human Niemann-Pick type C disease. J Biol Chem 278:32569-32577.
Cross DA, Alessi DR, Cohen P, Andjelkovich M, Hemmings BA (1995) Inhibition of glycogen synthase kinase-3 by insulin mediated by protein kinase B. Nature 378:785-789.

DeMattos RB (2004) Apolipoprotein E dose-dependent modulation of beta-amyloid deposition in a transgenic mouse model of Alzheimer's disease. J Mol Neurosci 23:255-262.

DeMattos RB, Cirrito JR, Parsadanian M, May PC, O’Dell MA, Taylor JW, Harmony JA, Aronow BJ, Bales KR, Paul SM, Holtzman DM (2004) ApoE and clusterin cooperatively suppress Abeta levels and deposition. Evidence that apoE regulates extracellular Abeta metabolism in vivo. Neuron 41:193-202.

Dietschy JM, Turley SD (2004) Cholesterol metabolism in the central nervous system during early development and in the mature animal. J Lipid Res 45:1375-1397.

Fagan AM, Murphy BA, Patel SN, Kilbridge JF, Mobley WC, Bu G, Holtzman DM (1998) Evidence for normal aging of the septo-hippocampal cholinergic system in apoE $(-/-)$ mice but impaired clearance of axonal degeneration products following injury. Exp Neurol 151:314-325.

Goedert M, Spillantini MG (2006) A century of Alzheimer's disease. Science 314:777-781.

Gong JS, Kobayashi M, Hayashi H, Zou K, Sawamura N, Fujita SC, Yanagisawa K, Michikawa M (2002) Apolipoprotein E (ApoE) isoformdependent lipid release from astrocytes prepared from human ApoE3 and ApoE4 knock-in mice. J Biol Chem 277:29919-29926.

Han X (2004) The role of apolipoprotein E in lipid metabolism in the central nervous system. Cell Mol Life Sci 61:1896-1906.

Harris-White ME, Frautschy SA (2005) Low density lipoprotein receptorrelated proteins (LRPs), Alzheimer's and cognition. Curr Drug Targets CNS Neurol Disord 4:469-480.

Hayashi H, Campenot RB, Vance DE, Vance JE (2004) Glial cell lipoproteins stimulate axon growth of central nervous system neurons in compartmented cultures. J Biol Chem 279:14009-14015.

Herz J, Bock HH (2002) Lipoprotein receptors in the nervous system. Annu Rev Biochem 71:405-434.

Herz J, Chen Y (2006) Reelin, lipoprotein receptors and synaptic plasticity. Nat Rev Neurosci 7:850-859.

Herz J, Strickland DK (2001) LRP: a multifunctional scavenger and signaling receptor. J Clin Invest 108:779-784.

Hirsch-Reinshagen V, Zhou S, Burgess BL, Bernier L, McIsaac SA, Chan JY, Tansley GH, Cohn JS, Hayden MR, Wellington CL (2004) Deficiency of ABCA1 impairs apolipoprotein E metabolism in brain. J Biol Chem 279:41197-41207.

Holtzman DM, Pitas RE, Kilbridge J, Nathan B, Mahley RW, Bu G, Schwartz AL (1995) Low density lipoprotein receptor-related protein mediates apolipoprotein E-dependent neurite outgrowth in a central nervous system-derived neuronal cell line. Proc Natl Acad Sci USA 92:9480-9484.

Huang Z, Hou Q, Cheung NS, Li QT (2006) Neuronal cell death caused by inhibition of intracellular cholesterol trafficking is caspase dependent and associated with activation of the mitochondrial apoptosis pathway. J Neurochem 97:280-291.

Ignatius MJ, Gebicke-Harter PJ, Skene JH, Schilling JW, Weisgraber KH, Mahley RW, Shooter EM (1986) Expression of apolipoprotein E during nerve degeneration and regeneration. Proc Natl Acad Sci USA 83:1125-1129.

Innerarity TL, Pitas RE, Mahley RW (1979) Binding of arginine-rich (E) apoprotein after recombination with phospholipid vesicles to the low density lipoprotein receptors of fibroblasts. J Biol Chem 254:4186-4190.

Kounnas MZ, Argraves WS, Strickland DK (1992) The 39-kDa receptorassociated protein interacts with two members of the low density lipoprotein receptor family, alpha 2-macroglobulin receptor and glycoprotein 330. J Biol Chem 267:21162-21166.

Kounnas MZ, Chappell DA, Strickland DK, Argraves WS (1993) Glycoprotein 330 , a member of the low density lipoprotein receptor family, binds lipoprotein lipase in vitro. J Biol Chem 268:14176-14181.

Kuo G, Arnaud L, Kronstad-O'Brien P, Cooper JA (2005) Absence of Fyn and Src causes a reeler-like phenotype. J Neurosci 25:8578-8586.

Li X, Kan HY, Lavrentiadou S, Krieger M, Zannis V (2002) Reconstituted discoidal ApoE-phospholipid particles are ligands for the scavenger receptor BI. The amino-terminal 1-165 domain of ApoE suffices for receptor binding. J Biol Chem 277:21149-21157.

Linseman DA, Butts BD, Precht TA, Phelps RA, Le SS, Laessig TA, Bouchard RJ, Florez-McClure ML, Heidenreich KA (2004) Glycogen synthase 
kinase- $3 \beta$ phosphorylates Bax and promotes its mitochondrial localization during neuronal apoptosis. J Neurosci 24:9993-10002.

Mabile L, Lefebvre C, Lavigne J, Boulet L, Davignon J, Lussier-Cacan S, Bernier L (2003) Secreted apolipoprotein E reduces macrophagemediated LDL oxidation in an isoform-dependent way. J Cell Biochem 90:766-776.

Mahley RW (1988) Apolipoprotein E: cholesterol transport protein with expanding role in cell biology. Science 240:622-630.

Masliah E, Mallory M, Ge N, Alford M, Veinbergs I, Roses AD (1995) Neurodegeneration in the central nervous system of apo E-deficient mice. Exp Neurol 136:107-122.

Mauch DH, Nagler K, Schumacher S, Goritz C, Muller EC, Otto A, Pfrieger FW (2001) CNS synaptogenesis promoted by glia-derived cholesterol. Science 294:1354-1357.

Mora A, Sabio G, Gonzalez-Polo RA, Cuenda A, Alessi DR, Alonso JC, Fuentes JM, Soler G, Centeno F (2001) Lithium inhibits caspase 3 activation and dephosphorylation of PKB and GSK3 induced by K+ deprivation in cerebellar granule cells. J Neurochem 78:199-206.

Murer MG, Yan Q, Raisman-Vozari R (2001) Brain-derived neurotrophic factor in the control human brain, and in Alzheimer's disease and Parkinson's disease. Prog Neurobiol 63:71-124.

Noble W, Planel E, Zehr C, Olm V, Meyerson J, Suleman F, Gaynor K, Wang L, LaFrancois J, Feinstein B, Burns M, Krishnamurthy P, Wen Y, Bhat R, Lewis J, Dickson D, Duff K (2005) Inhibition of glycogen synthase kinase- 3 by lithium correlates with reduced tauopathy and degeneration in vivo. Proc Natl Acad Sci USA 102:6990-6995.

Petegnief V, Saura J, de Gregorio-Rocasolano N, Paul SM (2001) Neuronal injury-induced expression and release of apolipoprotein $\mathrm{E}$ in mixed neuron/glia co-cultures: nuclear factor kappaB inhibitors reduce basal and lesion-induced secretion of apolipoprotein E. Neuroscience 104:223-234.

Ranganathan S, Liu CX, Migliorini MM, Von Arnim CA, Peltan ID, Mikhailenko I, Hyman BT, Strickland DK (2004) Serine and threonine phosphorylation of the low density lipoprotein receptor-related protein by protein kinase Calpha regulates endocytosis and association with adaptor molecules. J Biol Chem 279:40536-40544.
Ruiz J, Kouiavskaia D, Migliorini M, Robinson S, Saenko EL, Gorlatova N, Li D, Lawrence D, Hyman BT, Weisgraber KH, Strickland DK (2005) The apoE isoform binding properties of the VLDL receptor reveal marked differences from LRP and the LDL receptor. J Lipid Res 46:1721-1731.

Segal RA, Greenberg ME (1996) Intracellular signaling pathways activated by neurotrophic factors. Annu Rev Neurosci 19:463-489.

Sheng H, Laskowitz DT, Mackensen GB, Kudo M, Pearlstein RD, Warner DS (1999) Apolipoprotein E deficiency worsens outcome from global cerebral ischemia in the mouse. Stroke 30:1118-1124.

Siegel GJ, Chauhan NB (2000) Neurotrophic factors in Alzheimer's and Parkinson's disease brain. Brain Res Brain Res Rev 33:199-227.

Strittmatter WJ, Saunders AM, Schmechel D, Pericak-Vance M, Enghild J, Salvesen GS, Roses AD (1993) Apolipoprotein E: high-avidity binding to $\beta$-amyloid and increased frequency of type 4 allele in late-onset familial Alzheimer's disease. Proc Natl Acad Sci USA 90:1977-1981.

Su Y, Ryder J, Li B, Wu X, Fox N, Solenberg P, Brune K, Paul S, Zhou Y, Liu F, Ni B (2004) Lithium, a common drug for bipolar disorder treatment, regulates amyloid-beta precursor protein processing. Biochemistry 43:6899-6908.

Sun X, Sato S, Murayama O, Murayama M, Park JM, Yamaguchi H, Takashima A (2002) Lithium inhibits amyloid secretion in COS7 cells transfected with amyloid precursor protein C100. Neurosci Lett 321:61-64.

Thuahnai ST, Lund-Katz S, Williams DL, Phillips MC (2001) Scavenger receptor class B, type I-mediated uptake of various lipids into cells. Influence of the nature of the donor particle interaction with the receptor. J Biol Chem 276:43801-43808.

Wilkinson SE, Parker PJ, Nixon JS (1993) Isoenzyme specificity of bisindolylmaleimides, selective inhibitors of protein kinase C. Biochem J 294:335-337.

Yuan J, Yankner BA (2000) Apoptosis in the nervous system. Nature 407:802-809.

Zhuo M, Holtzman DM, Li Y, Osaka H, DeMaro J, Jacquin M, Bu G (2000) Role of tissue plasminogen activator receptor LRP in hippocampal longterm potentiation. J Neurosci 20:542-549. 